\title{
Identification of associated molecular events in Helicobacter pylori infection and gastric cancer through integrated computational analysis of Microarray and RNA-Seq datasets
}

\section{Sabaoon Zeb}

National University of Sciences and Technology (NUST), Research Centre for Modeling and Simulation (RCMS)

Rehan Zafar Paracha ( $\nabla$ rehan@rcms.nust.edu.pk)

National University of Sciences and Technology (NUST), Research Centre for Modeling and Simulation (RCMS)

\section{Maryum Nisar}

National University of Sciences and Technology (NUST), Research Centre for Modeling and Simulation (RCMS)

\section{Rimsha Khalid}

National University of Sciences and Technology (NUST), Research Centre for Modeling and Simulation (RCMS)

\section{Zartasha Mustansar}

National University of Sciences and Technology (NUST), Research Centre for Modeling and Simulation (RCMS)

\section{Samawia Rizwan}

National University of Sciences and Technology (NUST), Research Centre for Modeling and Simulation (RCMS)

\section{Saddia Bano}

National University of Sciences and Technology (NUST), Research Centre for Modeling and Simulation (RCMS)

\section{Research Article}

Keywords:

Posted Date: December 29th, 2021

DOI: https://doi.org/10.21203/rs.3.rs-1189424/v1 
License: (c) (i) This work is licensed under a Creative Commons Attribution 4.0 International License. Read Full License 


\title{
Identification of associated molecular events in Helicobacter pylori infection and gastric cancer through integrated computational analysis of Microarray and RNA-Seq datasets
}

\author{
Sabaoon Zeb ${ }^{1}$, Rehan Zafar Paracha ${ }^{1, *}$, Maryum Nisar ${ }^{1}$, Rimsha Khalid ${ }^{1}$, Zartasha \\ Mustansar $^{1}$, Samawia Rizwan ${ }^{1}$, and Saddia Bano ${ }^{1}$
}

${ }^{1}$ National University of Sciences and Technology (NUST), Research Centre for Modeling and Simulation (RCMS), Islamabad, 44000, Pakistan

*rehan@rcms.nust.edu.pk

\begin{abstract}
According to the World Health Organization, Gastric cancer (GC) is the third leading cause of death worldwide, where, the major precursor of cancer progression is infection with Helicobacter pylori. It has been reported that $50 \%$ of the total populace is infected with H.pylori, while in $80 \%$ the ulcer emerges in later stages of the infection. Although extensive separate analysis has been performed on H.pylori infection and GC data, however, there is a need to perform comparative analysis to identify the cross-talk between the conditions and to hunt significant molecular events that occurs during H.pylori induced GC. The aim of this multi-population study was to identify common molecular events and potential bio-markers against $H$.pylori induced GC. We performed microarray and RNA-seq analysis on publicly available H.pylori infection, gastritis, H.pylori induced GC and GC datasets to obtain Differentially Expressed Genes (DEGs). After obtaining the DEGs, integrative analysis, functional enrichment analysis and network biology approaches were utilized to identify common markers and hub genes between various disease conditions. Functional enrichment analysis revealed the DEGs of H.pylori infection, gastritis, H.pylori induced GC and GC were strongly associated with spliceosome, adherens junction, focal adhesion and ribosome. Being one of the common DEG, and highly interactive hub protein in the networks of all the conditions, translationally controlled tumour protein (TPT1) was identified as a significant predictive biomarker for early prognosis and diagnosis of H.pylori induced GC. Therefore, the mechanisms behind TPT1 should be further studied using in vitro cell-based functional assays, to determine its role in the progression of H.pylori induced GC.
\end{abstract}

\section{Introduction}

Helicobacter pylori (H.pylori), a gram-negative and spiral-shaped bacteria, is a pathogen that resides in the gastric epithelium ${ }^{1}$. Based on poor hygienic conditions in the developing countries, almost $80 \%$ of the people having ulcer is due to the H.pylori infection $^{2}$. Due to the asymptomatic nature of H.pylori, the infection is not timely diagnosed and if it is not treated on time the infection slowly progress towards gastric ulcer which further leads towards gastric cancer (GC) ${ }^{3}$. GC is the fifth commonly diagnosed cancer, whereas, it is the third leading cause of death worldwide ${ }^{4}$. Although, several socio-economic, environmental, molecular and genetic factors are involved in the progression of GC, however, one of the major culprit behind gastric carcinoma is H.pylori ${ }^{5,6}$.

Virulence factors produced during pathogenesis of H.pylori that are responsible for gastric carcinoma are cytotoxinassociated gene A (CagA), vacuolating cytotoxin (VacA) and type 4 secretory system (T4SS) along with outer inflammatory protein $\mathrm{A}(\mathrm{OipA})^{7}$. Where, some of the major host signalling pathways regulated or deregulated by $H$.pylori virulence factors include Jak/Stat signalling pathway, NFKB signalling pathway, focal adhesion, Src/MEK/ERK signalling pathway, autophagy pathway and c-Met-PI3K/Akt-mTOR signalling pathways, where these pathways has also found to be involved in the progression of $\mathrm{GC}^{8-11}$. Infection with H.pylori also instigates methylation of explicit genes; this explicitness is dictated by the epigenetic status in typical cells, including the occurrence of H3K27me 3 and RNA polymerase II. These specific sorts of aggravation i.e., driven by the H.pylori infection are significant for methylation, stimulation and invasion of monocytes and has all the earmarks of being involved in progression of $\mathrm{GC}^{12}$.

In previous studies, extensive analysis on H.pylori infection and GC datasets has been performed and the link between H.pylori infection and GC has been reported with the involvement of similar genes and pathways ${ }^{13-15}$. However, comparative analysis on H.pylori infection and GC samples from multi-population studies have not been performed previously by the integration of microarray and RNA-seq gene expression profiling. Therefore, it is necessary to perform a comparative analysis 
to describe the common and unique Differentially Expressed Genes (DEGs) between H.pylori infection, gastritis, H.pylori induced GC and GC. Further, functional enrichment and network analyses of the DEGs is a necessary step to acquire insights into the common molecular features that are involved in all the conditions to identify potential targets.

Based on multi-population data, we aim to analyse the progression of H.pylori pathogenesis towards GC by hunting common molecular events with an active role in the conditions. Differential expression analysis was performed on microarray and RNA-seq datasets of H.pylori infection, gastritis, H.pylori infected GC and GC to obtain significant DEGs. Functional enrichment analysis was performed to identify the role of the DEGs in various biological pathways and molecular functions. Furthermore, to unveil the relationship between proteins encoded by several genes in H.pylori infection and GC, and markers involved in the progression of H.pylori induced GC, network analysis was performed to identify the hub genes. The analysis revealed translationally controlled tumour protein (TPT1) to be a common marker and one of the highly interactive proteins in the H.pylori infection, gastritis, H.pylori infected GC and GC networks.

\section{Results}

The principal objective of this study was to identify linking molecular events, common patterns and key interactions in the different disease states i.e., H.pylori infection, gastritis, H.pylori infected GC and GC. For this purpose, we performed differential expression analysis on microarray and RNA-seq datasests followed by integrative, functional enrichment and network analyses.

\section{Differential expression Analysis}

Differential expression analysis on microarray and RNA-seq datasets was performed to obtain significant DEGs. The total number of DEGs obtained from microarray and RNA-seq analysis of H.pylori infection, gastritis,H.pylori infected GC and GC datasets are mentioned in Table 2. Moreover, significant DEGs were visualized through volcano plots, as shown in Figure 2 and 3 , which also resulted in visualization of the up-regulated and down-regulated DEGs.

\section{Comparative analysis}

For the identification of common biomarkers in H.pylori infection, gastritis, H.pylori infected GC datasets integrated analysis was performed. The results of the integrative analysis of the DEGs from microarray datasets identified 4 common genes (BIRC3,NAMPT,MMP1,ACSM3) in H.pylori infection whereas 119 common genes in GC datasets (Figure 4). Moreover when compared, no common markers were identified in the microarray H.pylori infection and GC datasets. Similarly, the DEGs from RNA-seq H.pylori infection, gastritis, H.pylori infected GC and GC datasets were then integrated, which identified 222 common genes in H.pylori infection and H.pylori infected GC datasets and 13 common genes in GC datasets (Figure 5). After comparing DEGs of all the four conditions, 2 genes (TPT1 and LYPD6B) were identified to be common in the RNA-seq H.pylori infection, gastritis, H.pylori infected GC and GC datasets.

\section{Functional Enrichment analysis}

Gene ontology (GO) and pathway analysis were performed to identify the biological processes and molecular pathways involved in H.pylori infection, gastritis, H.pylori infected GC and GC RNA-seq datasets. From the Toppfun function of Toppgene, the top GO results for H.pylori infection, gastritis, H.pylori infected GC and GC DEGs are mentioned in Table 3. Information mentioned in the table are the GO IDs, functions the DEGs are involved in i.e. molecular function (MF), biological process (BP) and cellular components (CC), significant p.value, false discovery rate (FDR) and the entities represents the DEGs involved in a particular function. The genetic ontologies revealed the involvement of the significant DEGs in molecular binding, RNA binding, cell adhesion molecular binding, cadherin binding and macromolecular junctions and complexes.

Pathway analysis of the DEGs performed through DAVID revealed significant associations and roles of the genes in various similar biological mechanisms. Furthermore, Cytoscape was used to visually represent the common interactive pathways involved in H.pylori infection, gastritis, H.pylori infected GC and GC as shown in Figure 6. Spliceosome was found to be the common pathway involved in all the conditions, whereas, adherens junction and RNA transport were the most commonly interactive pathways.

Spliceosome, being highly significant pathway identified in all the conditions was further explored to find out common markers across the conditions. HNRNPC, DDX42 and HNRNPU were the differentially expressed common entities found in H.pylori infection, gastritis H.pylori infected GC and GC. Moreover, other pathways that were enriched in the respective conditions included ribosome, adherens junction, focal adhesion and Alzheimer's disease and RNA transport. The top enriched pathways involved in H.pylori infection, gastritis, H.pylori infected GC and GC are mentioned in Table 4.

\section{Network analysis and Hub nodes identification}

To identify the hub genes and protein-protein interaction (PPI) networks of H.pylori infection, gastritis, H.pylori infected GC and GC DEGs, or between the conditions, network and module analysis was performed. PPI networks were identified using the 
STRING database with the highest confidence score (0.9) which was further modified and visualized by Cytoscape software along with module analysis. The PPI analysis indicated 171 genes in H.pylori infection, 112 genes in gastritis, 1105 genes in H.pylori infected GC and 183 genes in GC that were found to be differentially expressed in the respective conditions. After analyzing the networks of H.pylori infection, gastritis, H.pylori infected GC and GC, hub nodes were identified with the highest degree of interactions. These PPI networks were further merged to evaluate the genomic connectivity within the neighboring genes along with common interactions. Network analysis of the H.pylori infection network revealed 17 hub genes (RPS20, RPLP0, RPS17, SRSF3, RPL8, POLR2L, SENP3, RPL6, SRRT, RNPS1, RPL39, TRA2B, SLU7, SRRM2, RPL23, RPL35A and RPL32) based on the degree values as shown in plot A of Figure 7 and Table 5.

To identify the core modules, the MCODE app in Cytoscape was used to find the highly connected nodes in the H.pylori infection network with Node Score Cutoff: 0.2 Haircut: true Fluff: false and K-Core: 2. In the network, 31 clusters were generated in which 1 cluster was selected based on the highest scores and highly connected nodes shown in plot B of Figure 7 having 32 nodes, 272 edges and a 17.5 density score. Similarly, network analysis revealed 9 hub genes (SRSF7, U2AF2, ITGB1, SF1, TRIM21, HIST1H2AH, RPL6, UBE2G2 and RAP1B) in the gastritis dataset with the highest degree of interaction as shown in plot $\mathrm{E}$ of Figure 7. MCODE analysis resulted in 10 clusters out of which 1 highly interactive cluster as shown in plot F of Figure 7 with 9 nodes, 35 edges and an 8.5 density score was selected.

In the network of H.pylori infected GC, 82 hub nodes were identified of which UBC, TP53, RPS7, RPS9, UBE2C, RPS4X, RPS3, RPS2, UBR4 and RPS26 were the top highly connected nodes as shown in Figure 7 based on the degree as shown in Table 5. Module analysis formed 43 clusters out of which 1 highly connected cluster was selected from the network having 103 nodes, 2541 edges and 49.8 a density score. Whereas, in the GC network, 9 hub genes (RPL13, RPL11, RPL37A, RPLP0, RPL34, RPL32, RPS15A, RPL3 and RPS12) were selected as shown in plot C of Figure 7, based on the degree as mentioned in Table 5 and high connectivity with the neighboring nodes. After MCODE analysis, 9 clusters were obtained of which 1 cluster was selected as shown in plot D of Figure 7 that depicted high connection within the network of GC having 17 nodes, 128 edges and a density score of 16 . Average shortest path length, betweenness centrality, closeness centrality, clustering coefficient, degree, eccentricity and neighbourhood connectivity for all the hub genes of H.pylori infection, gastritis, H.pylori infected GC and GC are mentioned in Table 5.

\section{Network summary statistics}

The summary statistics of H.pylori infection, gastritis, H.pylori infected GC and GC networks were obtained using the network analyzer tool in Cytoscape. It provided statistics regarding the number of nodes and edges, connected components, average number of neighbours and clustering coefficient in PPI networks as mentioned in Table 6.

\section{Common interactions}

To identify common genetic markers between significant DEGs of the four dense networks i.e. H.pylori infection, gastritis, H.pylori infected GC and GC, nodes from the four PPI networks were merged. In the merged PPI network, the nodes of H.pylori infection are given red color, gastritis indicates the green color, H.pylori infected GC are represented in purple color and GC is given dark blue color in Figure 8. Highlighted as the yellow node, in the merged PPI network TPT1 was identified to be the common interaction in all of the four conditions. Whereas, LMO7, RAP1B and RPL6 were the 3 common interactions between H.pylori infection, gastritis and H.pylori infected GC that are shown as pink nodes in Figure 8. Moreover, the light blue nodes (RPLP0, RPL32 and SRRM2) accentuates the highly connected common interactions in H.pylori infection, H.pylori infected GC and GC. Ultimately, ITGB1 is identified as the common interaction between gastritis, H.pylori infected GC and $\mathrm{GC}$ and is shown as an orange node in the network in Figure 8.

According to the study, low expression of TPT1 was observed in H.pylori infection (GSE55699) $\left(\log _{2} \mathrm{FC}=-20.07\right)$ and GC (GSE41476, GSE80388) $\left(\log _{2} \mathrm{FC}=-3.28, \log _{2} \mathrm{FC}=-3.42\right)$ datasets, whereas, it was found to be highly expressed in H.pylori infected GC (GSE73226) $\left(\log _{2} \mathrm{FC}=5.79\right)$ and gastritis (E-MTAB-3689) $\left(\log _{2} \mathrm{FC}=1.9\right)$ datasets. The gradual increase in the expression patterns of TPT1 at various stages i.e. H.pylori infection, gastritis and H.pylori infected GC suggests that TPT1 may have a significant role in the progression of $\mathrm{H}$. pylori induced cancer.

\section{Discussion}

According to GLOBOCAN's cancer statistics, worldwide, Gastric cancer (GC) is reported to be the third leading cause of death ${ }^{16}$, where, one of the major causes of carcinoma is Helicobacter pylori infection ${ }^{17}$. The pathogen asymptomatically resides in the stomach for years and in later stages progress into GC due to numerous H.pylori virulence factors i.e. CagA, VacA, T4SS etc ${ }^{18}$. To prevent this silent progression, early antibiotic treatment of H.pylori infection is required, unfortunately, the pathogen is showing resistance against major antibiotics including Clarithromycin, Metronidazole, Levofloxacin, Amoxicillin, Tetracyclin, Rifabutin and Furazolidon ${ }^{19}$. Therefore it is crucial to identify key essential genes and discover common biomarkers that might have a potential role in H.pylori infected GC. The advancements in computational approaches and experimental 
technologies have opened up new possibilities for the scientific community to explore the role of the gut microbiome in tumor microenvironment on molecular, genetic and epigenetic levels ${ }^{20}$.

Previously, scientists have performed differential expression analysis on H.pylori infection and GC datasets including microarray and RNA-seq analysis ${ }^{13-15}$. However, these studies have generated, analyzed and explored data of a targeted population to understand the genetic variations in H.pylori infection and GC. A multi-population integrative data analysis has not been performed to investigate the cross-talk between H.pylori infection and GC. Therefore, it is crucial to perform a multi-population differential expression based study to identify genetic biomarkers and common interactions between $H$.pylori infection and GC.

This study explored the differential expression in H.pylori infection, gastritis, H.pylori infected GC and GC by analyzing microarray and RNA-seq datasets and resulted in significant differentially expressed genes (DEGs) of the respective conditions. Based on the availability of data, the countries selected for this multi-population differential expression study were Hungary, Portugal, China, Germany, South Korea, Taiwan and Nicaragua (Central America). Once the significant DEGs were acquired from the selected datasets (GSE55699 ${ }^{21}$, GSE73226 ${ }^{22}$, GSE41476 $6^{23}$, GSE80388 24 and E-MTAB-3689 ${ }^{25}$ ), functional enrichment and network analyses were carried out to identify potential genetic biomarkers and common interactions. We were not able to achieve commonality between the microarray and RNA-seq DEGs and the reason behind it might be due to the difference in both the approaches as well as selection of data from different populations.

Translationally controlled tumor protein (TPT1) and Ly6/Plaur Domain Containing 6b (LYPD6B) were identified as the common biomarkers for H.pylori induced GC. Till now, LYPD6B is only identified as a potential biomarker in the progression of ovarian cancer $^{26}$ and is not studied in other cancers including GC. Although LYPD6B was one of the common DEGs, however, no interactions were identified with the other DEGs. Being differentially expressed in all the conditions and showing a high degree of connectivity with other interacting genes, TPT1 (p.value $<0.05$ ) was identified as a potential biomarker, based on the comparative and network analyses. It has been reported that TPT1 has an essential role in various biological processes including cell growth and survival, cell proliferation, DNA damage and repair, cellular reprogramming, inflammation and biogenesis of ribosomes, RNA and proteins ${ }^{27,28}$. High expression of TPT1 is proved to have malignancy promoting characteristic in lung, colon and prostate cancers, where, TPT1 is found to be involved in cancer development and progression ${ }^{27,28}$. Similarly, in gastric carcinoma high expression of TPT1 has revealed its involvement in the later stages of malignancy i.e. invasion and metastasis ${ }^{29,30}$.

The common pathway where DEGs of H.pylori infection, gastritis, H.pylori infected GC and GC appeared to be enriched was spliceosome where the three common identified biomarkers in all the conditions were Heterogeneous nuclear ribonucleoproteins C1/C2 (HNRNPC), DEAD-Box Helicase 42 (DDX42) and Heterogeneous nuclear ribonucleoprotein U (HNRNPU). Spliceosome genes are found to be involved in the progression of various cancers including $\mathrm{GC}^{31-33}$, however, its role in H.pylori induced GC is not explored. The other significant pathways were ribosome, adherens junction, Alzheimer's disease and focal adhesion that are previously reported to be involved in H.pylori induced GC ${ }^{34-37}$. Alzheimer's disease was one of the common pathways in H.pylori infection and GC datasets where Amyloid precursor protein (APP), Cytochrome C Oxidase Subunit 4I1 (COX4I1), ATPase Sarcoplasmic/Endoplasmic Reticulum Ca2+ Transporting 3 (ATP2A3), Caspase-9 (CASP9) and Caspase-7 (CASP7) were identified as the common DEGs. Although H.pylori infection is reported to be one of the causative agents of Alzheimer's disease ${ }^{38,39}$, however, its role in H.pylori induced GC is yet to be explored. To study the connection between Alzheimer's disease and H.pylori induced GC APP, COX4I1, ATP2A3, CASP9 and CASP7 should be studied to get insights into the molecular functions involved in the process.

Moreover, protein-protein interaction (PPI) networks revealed interaction of TPT1 with several 60S subunit ribosomal proteins including ribosomal Protein Lateral Stalk Subunit P0 (RPLP0), ribosomal protein L32 (RPL32), ribosomal protein L11 (RPL11), ribosomal protein L12 (RPL12), ribosomal protein L24 (RPL24) and ribosomal protein L25A (RPL25A). Although ribosomal proteins have a significant role in the progression of several cancers including colorectal cancer ${ }^{40}$, breast cancer ${ }^{41}$, prostate cancer ${ }^{42}$, lymphoblastic leukaemia ${ }^{43}$ and cervical cancer ${ }^{44}$. However, to the best of our knowledge and literature search, the role of ribosomal proteins in H.pylori induced GC has not been explored yet. LIM domain only protein 7 (LMO7), Ras-related Protein Rap-1b (RAP1B), Ribosomal Protein L6 (RPL6), RPLP0, RPL32, Serine/arginine repetitive matrix protein 2 (SRRM2) and Integrin Subunit Beta 1 (ITGB1) were identified as other common potential interactions. The hub genes in H.pylori infection, gastritis, H.pylori infected GC and GC PPI networks were selected based on the degree of connectivity where ribosomal genes were found to be involved in all the above conditions except gastritis. Which shows that in several ways ribosomal proteins contribute to the progression of H.pylori induced GC and it is crucial to study their role.

\section{Conclusion}

In conclusion, comparative analysis identified translationally controlled tumor protein (TPT1) and Ly6/Plaur Domain Containing $6 \mathrm{~b}$ (LYPD6B) to be the common DEGs across all the RNA-seq datasets. Overall, the most significant pathways where the genes were found to be enriched were spliceosome, focal adhesion, adherens junction and Alzheimer's disease. Network analysis 
helped us identify TPT1 as a potential predictive biomarker for H.pylori induced GC due to its presence and highly interactive nature across the networks of all the conditions. In future, to better understand the role of TPT1 in H.pylori induced GC, this study needs to be further validated experimentally by using in vitro cell-based functional assays, whereas, TPT1 can be targeted by using inhibitors and enhancers.

\section{Methods}

The differential expression analysis of microarray and RNA-seq datasets was performed followed by functional enrichment and network analyses for identification of linking molecular events and significant biomarkers. The overall methodology performed on the datasets of H.pylori infection and GC is shown in Figure 1.

\section{Data collection}

In this study, a total of 11 datasets for Microarray and RNA-seq analysis were retrieved from ArrayExpress ${ }^{45}$ and Gene Expression Omnibus (GEO) ${ }^{46}$ databases. For the H.pylori induced GC datasets, the selection criterias were the organism to be Homo sapiens with normal and disease conditions, the samples being treatment-naive i.e no effect of drugs on the samples and no mutation in the genes in the datasets. Based upon our inclusion criteria, the selected datasets belonged to different regions which ended up making our research a multi-population study, which ended up removing geographic region bias from our analysis. Details of ID, platform, number of samples, the origin of the microarray (i.e. H.pylori infection and GC), RNA-seq (i.e. H.pylori infection, gastritis, H.pylori infected GC and GC) datasets and the literature references are listed in Table 1.

\section{Data processing}

\section{Microarray data analysis}

Microarray data analysis on H.pylori infection and GC datasets was performed using end to end workflow ${ }^{47}$ in $\mathrm{R}^{48}$. Different Bioconductor R packages were used i.e Biobase, oligoClasses, ArrayExpress, oligo, arrayQualityMatrics, RMA ${ }^{49}$, limma ${ }^{50}$, and EnhancedVolcano ${ }^{51}$. Expression data from all the datasets were imported using ArrayExpress package that fetched ADF, IDF, SDRF and raw data files. Quality control was carried out on H.pylori infection and GC datasets. Normalization was then performed to remove the variation and outliers in the raw expression data using the Robust Multiarray average (RMA) package which performed background correction, normalization and summarization. Quality control and normalization were performed for both alignment and control followed by differential expression analysis using limma package to retrieve DEGs. For H.pylori infection and GC datasets, significant threshold (cut-off) negative log of p.value $<0.05$ and a $\log _{2}$ fold change $\left(\log _{2} \mathrm{FC}\right)\left(\log _{2} \mathrm{FC}<-1 \& \log _{2} \mathrm{FC}>1\right)$ were set to extract genes that were significantly differentially expressed. Moreover, for visualization of the DEGs, volcano-plot was generated by using the EnhancedVolcano package.

\section{RNA-seq data analysis}

A well-annotated pipeline was created and RNA-seq data analysis was carried out using Galaxy ${ }^{52}$ platform and R. Various tools in Galaxy were used to analyse the RNA-seq datasets including H.pylori infection, gastritis, H.pylori infected GC and GC datasets. Quality control was performed using the FastQC ${ }^{53}$ tool on the raw sequence data of RNA-Seq datasets to identify the adapter contents which were further removed using FASTQ Quality Trimmer ${ }^{54}$. The sequence reads were then aligned to the reference genome of Homo sapien i.e. Hg38 (present by default) using HISAT2 ${ }^{55}$. Moreover, to check the quality of data and identify sequence and mapping based duplicate reads $\mathrm{RSeQC}^{56}$ tool was used. The duplicate reads were then marked using MarkDuplicate Picard and further removed by the RmDup ${ }^{57}$ tool.

After the alignment, gene expression data were retrieved using StringTie ${ }^{58}$. The retrieved output files were assembled transcripts, exon, intron and transcript-level expression data, along with exon to transcript mapping and intron to transcript mapping files. For differential expression analysis, Ballgown ${ }^{58} \mathrm{R}$ script was used, which generated significant DEGs by processing the read count data obtained from StringTie. The significant DEGs of H.pylori infection, gastritis, H.pylori infected GC and GC datasets were then visualized using EnhancedVolcano plot with a cut-off p.value $<0.05$ and $\log _{2} \mathrm{FC}<-1$ and $\log _{2} \mathrm{FC}>1$. The aim of keeping similar $\log _{2} \mathrm{FC}$ values was to obtain a significant amount of DEGs and to make it comparable in further comparative, functional enrichment and network analyses.

\section{Comparative Analysis}

For identification of common markers and key genes, biomaRt was used to perform comparative analysis on the DEGs obtained through the differential expression analysis. To visualize the common DEGs between all the datasets InteractiVenn ${ }^{59}$ was used.

\section{Functional Enrichment and Network Analysis}

Gene Ontology (GO) and pathway analysis was performed to identify the biological and molecular aspects of DEGs involved in the progression of H.pylori infection, gastritis, H.pylori infected GC and GC. Ontologies were obtained using the Toppfun 
function in Toppgene ${ }^{60}$, and pathway analysis was performed using the DAVID ${ }^{61}$ database, where the KEGG (Kyoto Encyclopedia of Genes and Genomes) ${ }^{62}$ pathways were considered. Furthermore, common pathways in H.pylori infection, gastritis, H.pylori infected GC and GC datasets were selected with a p.value $<0.05$.

To further identify the interactions and hub genes between the DEGs of H.pylori infection, gastritis, H.pylori infected GC and GC conditions, network analysis was performed. To get an interacting network of each condition, the lists of the DEGs were uploaded to the STRING database (version: 11.0) ${ }^{63}$, where, the threshold for interaction score was set to have the highest confidence score of 0.9. For detailed network analysis, Cytoscape software (version: 3.8.2) ${ }^{64}$ was used for visualization and identification of highly dense clusters in the networks. Ultimately, to identify the interconnected nodes (DEGs) in the H.pylori infection, gastritis, H.pylori infected GC and GC networks and to understand their functionality, network analyzer ${ }^{65}$ tool and $^{\circ}$ $\mathrm{MCODE}^{66}$ app were utilized.

\section{References}

1. Ansari, S. \& Yamaoka, Y. Current understanding and management of helicobacter pylori infection: an updated appraisal. F1000Research 7, 721 (2018).

2. Owyang, S. Y. et al. Dendritic cell-derived tgf- $\beta$ mediates the induction of mucosal regulatory t-cell response to helicobacter infection essential for maintenance of immune tolerance in mice. Helicobacter 25, e12763 (2020).

3. Mégraud, F., Bessède, E. \& Varon, C. Helicobacter pylori infection and gastric carcinoma. Clin. Microbiol. Infect. 21, 984-990 (2015).

4. Tan, M. C., Balakrishnan, M. \& Graham, D. Y. Gastric cancer worldwide except japan. In Gastric Cancer, vol. 1, 17-28 (2019).

5. Liou, J.-M. et al. Screening and eradication of helicobacter pylori for gastric cancer prevention: the taipei global consensus. Gut 69, 2093-2112 (2020).

6. Chen, X. et al. Variation in gene expression patterns in human gastric cancers. Mol. biology cell 14, 3208-3215 (2003).

7. Chang, W.-L., Yeh, Y.-C. \& Sheu, B.-S. The impacts of h. pylori virulence factors on the development of gastroduodenal diseases. J. biomedical science 25, 68 (2018).

8. Krzysiek-Maczka, G. et al. Involvement of epithelial-mesenchymal transition-inducing transcription factors in the mechanism of helicobacter pylori-induced fibroblasts activation. J. Physiol. Pharmacol 70, 727-736 (2019).

9. Yong, X. et al. Helicobacter pylori virulence factor caga promotes tumorigenesis of gastric cancer via multiple signaling pathways. Cell communication signaling 13, 1-13 (2015).

10. Abdullah, M. et al. Vaca promotes caga accumulation in gastric epithelial cells during helicobacter pylori infection. Sci. reports $9,1-9$ (2019).

11. Li, N. et al. Helicobacter pylori caga protein negatively regulates autophagy and promotes inflammatory response via c-met-pi3k/akt-mtor signaling pathway. Front. cellular infection microbiology 7, 417 (2017).

12. Ushijima, T. \& Hattori, N. Molecular pathways: Involvement of helicobacter pylori-triggered inflammation in the formation of an epigenetic field defect, and its usefulness as cancer risk and exposure markers. Clin. Cancer Res. 18, 923-929 (2012).

13. Liu, S. et al. Differentially expressed mrnas and their long noncoding rna regulatory network with helicobacter pyloriassociated diseases including atrophic gastritis and gastric cancer. BioMed research international 2020 (2020).

14. Li, Y., Bai, W. \& Zhang, X. Identifying heterogeneous subtypes of gastric cancer and subtype-specific subpaths of microrna-target pathways. Mol. Medicine Reports 17, 3583-3590 (2018).

15. Lozano-Pope, I., Sharma, A., Matthias, M., Doran, K. S. \& Obonyo, M. Effect of myeloid differentiation primary response gene 88 on expression profiles of genes during the development and progression of helicobacter-induced gastric cancer. BMC cancer 17, 133 (2017).

16. Bray, F. et al. Global cancer statistics 2018: Globocan estimates of incidence and mortality worldwide for 36 cancers in 185 countries. CA: a cancer journal for clinicians 68, 394-424 (2018).

17. Liou, J.-M., Lee, Y.-C., El-Omar, E. M. \& Wu, M.-S. Efficacy and long-term safety of h. pylori eradication for gastric cancer prevention. Cancers 11, 593 (2019).

18. Kakelar, H. M. et al. Pathogenicity of helicobacter pylori in cancer development and impacts of vaccination. Gastric Cancer 22, 23-36 (2019). 
19. Savoldi, A., Carrara, E., Graham, D. Y., Conti, M. \& Tacconelli, E. Prevalence of antibiotic resistance in helicobacter pylori: a systematic review and meta-analysis in world health organization regions. Gastroenterology 155, 1372-1382 (2018).

20. Meng, C., Bai, C., Brown, T. D., Hood, L. E. \& Tian, Q. Human gut microbiota and gastrointestinal cancer. Genomics, proteomics \& bioinformatics 16, 33-49 (2018).

21. Koeppel, M., Garcia-Alcalde, F., Glowinski, F., Schlaermann, P. \& Meyer, T. F. Helicobacter pylori infection causes characteristic dna damage patterns in human cells. Cell reports 11, 1703-1713 (2015).

22. Glowinski, F., Garcia-Alcalde, F., Okonechnikov, K. \& Meyer, T. F. Modulation of the host cell rna splicing program by the gastric pathogen helicobacter pylori. EMBnet. journal 19, 70-71 (2013).

23. Park, S.-M. et al. A known expressed sequence tag, bm742401, is a potent lincrna inhibiting cancer metastasis. Exp. \& molecular medicine 45, e31 (2013).

24. Hsieh, Y.-Y. et al. Upregulation of bone morphogenetic protein 1 is associated with poor prognosis of late-stage gastric cancer patients. BMC cancer 18, 508 (2018).

25. Thorell, K. et al. In vivo analysis of the viable microbiota and helicobacter pylori transcriptome in gastric infection and early stages of carcinogenesis. Infect. immunity 85, e00031-17 (2017).

26. Shoji, Y. et al. Over-expression of ly6/plaur domain containing 6b (lypd6b) in ovarian cancer. Gynecol Obstet. 1, 103 (2011).

27. Hudler, P., Repše, S., Juvan, R. \& Komel, R. A genomic approach to investigate expression profiles in slovenian patients with gastric cancer. Oncol. letters 2, 1003-1014 (2011).

28. Amson, R., Pece, S., Marine, J.-C., Di Fiore, P. P. \& Telerman, A. Tpt1/tctp-regulated pathways in phenotypic reprogramming. Trends cell biology 23, 37-46 (2013).

29. Lee, J.-H. Overexpression of humbug promotes malignant progression in human gastric cancer cells. Oncol. reports 19, 795-800 (2008).

30. Kočevar, N., Odreman, F., Vindigni, A., Grazio, S. F. \& Komel, R. Proteomic analysis of gastric cancer and immunoblot validation of potential biomarkers. World J. Gastroenterol. WJG 18, 1216 (2012).

31. Liu, C. et al. Systematic profiling of alternative splicing in helicobacter pylori-negative gastric cancer and their clinical significance. Cancer Cell Int. 20, 1-15 (2020).

32. Feng, H. et al. Identification and validation of critical alternative splicing events and splicing factors in gastric cancer progression. J. cellular molecular medicine 24, 12667-12680 (2020).

33. Eymin, B. Targeting the spliceosome machinery: A new therapeutic axis in cancer. Biochem. pharmacology 114039 (2020).

34. Costa, A. M., Leite, M., Seruca, R. \& Figueiredo, C. Adherens junctions as targets of microorganisms: a focus on helicobacter pylori. FEBS letters 587, 259-265 (2013).

35. Sharafutdinov, I., Backert, S. \& Tegtmeyer, N. Cortactin: a major cellular target of the gastric carcinogen helicobacter pylori. Cancers 12, 159 (2020).

36. $\mathrm{Hu}$, Y. et al. Analysis of key genes and signaling pathways involved in helicobacter pylori-associated gastric cancer based on the cancer genome atlas database and rna sequencing data. Helicobacter 23, e12530 (2018).

37. Wang, B., Zhang, J., Chen, S. \& Bie, M. Helicobacter pylori and gastrointestinal and neurological diseases: Study protocol of an umbrella review of systematic reviews and meta-analyses. Medicine $\mathbf{9 8}$ (2019).

38. Chang, Y.-P. et al. Eradication of helicobacter pylori is associated with the progression of dementia: a population-based study. Gastroenterol. research practice 2013 (2013).

39. Franceschi, F. et al. Microbes and alzheimer'disease: lessons from h. pylori and gut microbiota. Eur Rev Med Pharmacol Sci 23, 426-430 (2019).

40. Slimane, S. N. et al. Ribosome biogenesis alterations in colorectal cancer. Cells 9, 2361 (2020).

41. Ebright, R. Y. et al. Deregulation of ribosomal protein expression and translation promotes breast cancer metastasis. Science 367, 1468-1473 (2020).

42. Arthurs, C. et al. Expression of ribosomal proteins in normal and cancerous human prostate tissue. PLoS One 12, e0186047 (2017). 
43. De Keersmaecker, K. et al. Exome sequencing identifies mutation in cnot 3 and ribosomal genes rpl5 and rpl10 in t-cell acute lymphoblastic leukemia. Nat. genetics 45, 186-190 (2013).

44. Zhou, H. et al. Overexpression of ribosomal rna in the development of human cervical cancer is associated with rdna promoter hypomethylation. PLoS One 11, e0163340 (2016).

45. Athar, A. et al. Arrayexpress update-from bulk to single-cell expression data. Nucleic acids research 47, D711-D715 (2018).

46. Edgar, R., Domrachev, M. \& Lash, A. E. Gene expression omnibus: Ncbi gene expression and hybridization array data repository. Nucleic acids research 30, 207-210 (2002).

47. Klaus, B. \& Reisenauer, S. An end to end workflow for differential gene expression using affymetrix microarrays. F1000Research 5 (2016).

48. Team, R. C. R: A language and environment for statistical computing. dim (ca533) 1, 34 (2018).

49. Irizarry, R. A. et al. Exploration, normalization, and summaries of high density oligonucleotide array probe level data. Biostatistics 4, 249-264 (2003).

50. Ritchie, M. E. et al. limma powers differential expression analyses for rna-sequencing and microarray studies. Nucleic acids research 43, e47-e47 (2015).

51. Blighe, K. Enhancedvolcano: Publication-ready volcano plots with enhanced colouring and labeling. $r$ package version 1.2. 0 (2019).

52. Afgan, E. et al. The galaxy platform for accessible, reproducible and collaborative biomedical analyses: 2018 update. Nucleic acids research 46, W537-W544 (2018).

53. Andrews, S. et al. FastQC. Babraham Institute (2012).

54. Blankenberg, D. et al. Manipulation of fastq data with galaxy. Bioinformatics 26, 1783-1785 (2010).

55. Kim, D., Langmead, B. \& Salzberg, S. L. Hisat: a fast spliced aligner with low memory requirements. Nat. Methods 12 (2015).

56. Wang, L., Wang, S. \& Li, W. Rseqc: quality control of rna-seq experiments. Bioinformatics 28 (2012).

57. Li, H. et al. The sequence alignment/map format and samtools. Bioinformatics 25 (2009).

58. Pertea, M., Kim, D., Pertea, G. M., Leek, J. T. \& Salzberg, S. L. Transcript-level expression analysis of rna-seq experiments with hisat, stringtie and ballgown. Nat. protocols 11, 1650 (2016).

59. Heberle, H., Meirelles, G. V., da Silva, F. R., Telles, G. P. \& Minghim, R. Interactivenn: a web-based tool for the analysis of sets through venn diagrams. BMC bioinformatics 16, 1-7 (2015).

60. Chen, J., Bardes, E. E., Aronow, B. J. \& Jegga, A. G. Toppgene suite for gene list enrichment analysis and candidate gene prioritization. Nucleic acids research 37, W305-W311 (2009).

61. Dennis, G. et al. David: database for annotation, visualization, and integrated discovery. Genome biology 4, R60 (2003).

62. Kanehisa, M., Furumichi, M., Tanabe, M., Sato, Y. \& Morishima, K. Kegg: new perspectives on genomes, pathways, diseases and drugs. Nucleic acids research 45, D353-D361 (2016).

63. Szklarczyk, D. et al. The string database in 2017: quality-controlled protein-protein association networks, made broadly accessible. Nucleic acids research gkw937 (2016).

64. Lopes, C. T. et al. Cytoscape web: an interactive web-based network browser. Bioinformatics 26, 2347-2348 (2010).

65. Doncheva, N. T., Assenov, Y., Domingues, F. S. \& Albrecht, M. Topological analysis and interactive visualization of biological networks and protein structures. Nat. protocols 7, 670 (2012).

66. Cline, M. S. et al. Integration of biological networks and gene expression data using cytoscape. Nat. protocols 2, 2366 (2007).

67. Galamb, O. et al. Helicobacter pylori and antrum erosion-specific gene expression patterns: the discriminative role of cxcl13 and vcam1 transcripts. Helicobacter 13, 112-126 (2008).

68. Costa, A. M. et al. Helicobacter pylori activates matrix metalloproteinase 10 in gastric epithelial cells via egfr and erk-mediated pathways. The J. infectious diseases 213, 1767-1776 (2016).

69. Zhu, H. et al. Microarray analysis of long non-coding rna expression profiles in human gastric cells and tissues with helicobacter pylori infection. BMC medical genomics $\mathbf{8}, 84$ (2015). 
70. Wang, Q. et al. Upregulated inhba expression is associated with poor survival in gastric cancer. Med. oncology 29, 77-83 (2012).

71. Sun, T. et al. Tmeff2 deregulation contributes to gastric carcinogenesis and indicates poor survival outcome. Clin. Cancer Res. 20, 4689-4704 (2014).

72. Popovici, V. et al. Effect of training-sample size and classification difficulty on the accuracy of genomic predictors. Breast Cancer Res. 12, 1-13 (2010).

73. Consortium, M. et al. The microarray quality control (maqc)-ii study of common practices for the development and validation of microarray-based predictive models. Nat. biotechnology 28, 827 (2010).

\section{Author contributions statement}

Sabaoon Zeb and Rehan Z. Paracha conceived and designed the study. Sabaoon Zeb conducted the analysis and wrote the manuscript. All authors took part in analytical discussions and critical reviewing of the manuscript.

\section{Additional information}

Not applicable

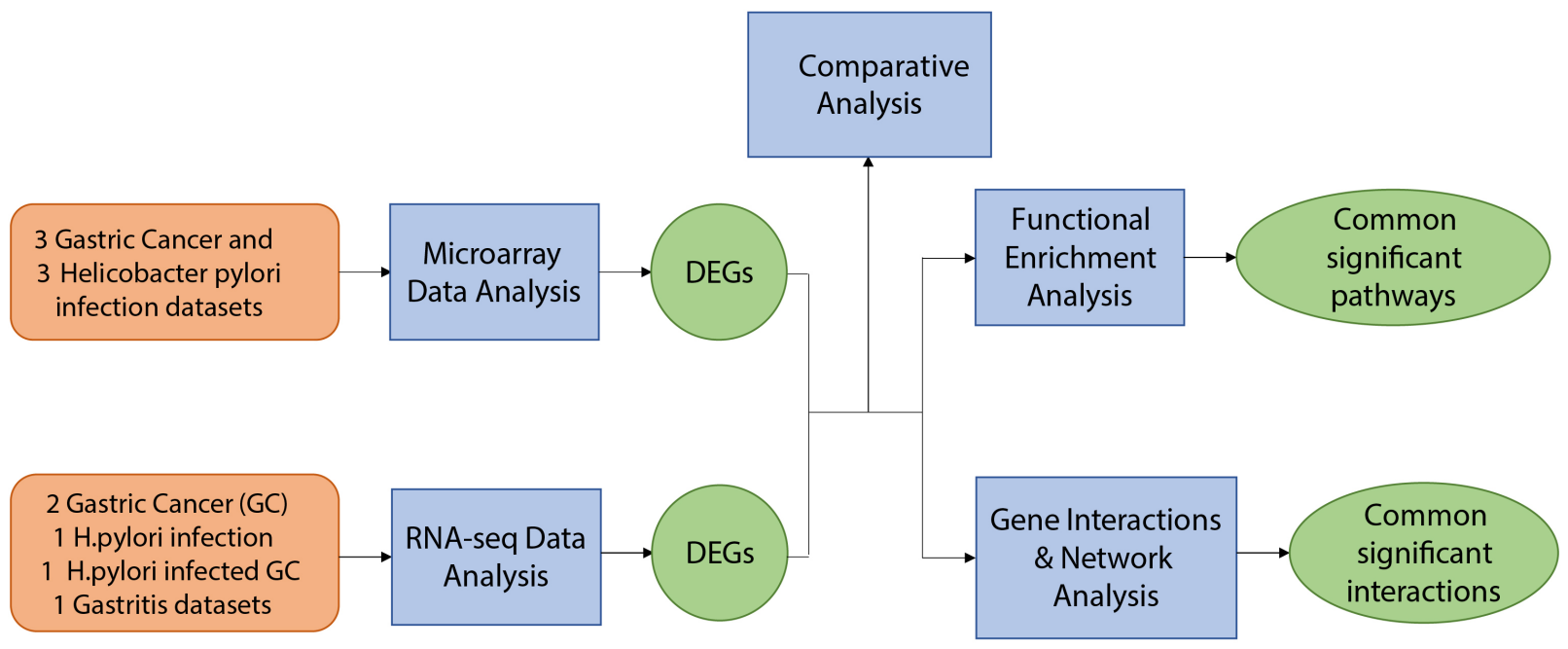

Figure 1. Overall methodology of the research Differential expression analysis was performed on microarray and RNA-seq datasets of H.pylori infection, gastritis, H.pylori infected GC and GC to obtain differentially expressed genes (DEGs). Further comparative analysis was performed to identify common genetic biomarkers followed by functional enrichment and network analysis for identification of common significant pathways and interactions. 
Table 1. Datasets information

\begin{tabular}{|c|c|c|c|c|}
\hline ID & Platform & Samples & Country & References \\
\hline \multicolumn{5}{|c|}{ Microarray Datasets } \\
\hline \multicolumn{5}{|c|}{ Helicobacter pylori infection } \\
\hline E-GEOD-5081 & Affymetrix [HG-U133Plus2] & 16 (8 non-infected \& 8 infected $)$ & Hungary & 67 \\
\hline E-GEOD-70394 & Agilent whole genome $4 \times 44 \mathrm{KV} 1$ & 6 (3 non-infected \& 3 infected) & Portugal & 68 \\
\hline E-GEOD-74577 & Affymetrix [HTA-20] & 6 (3 non-infected \& 3 infected $)$ & China & 69 \\
\hline \multicolumn{5}{|c|}{ Gastric cancer } \\
\hline E-GEOD-19826 & Affymetrix [HG-U133Plus2] & 27 (12 tumor \& 15 normal) & China & 70 \\
\hline E-GEOD-49051 & Agilent whole genome $4 \times 44 \mathrm{KV} 2$ & $6(3$ tumor $\& 3$ normal $)$ & China & 71 \\
\hline E-GEOD-79973 & Affymetrix [HG-U133Plus2] & 20 (10 tumor \& 10 normal $)$ & China & 72,73 \\
\hline \multicolumn{5}{|c|}{ RNA-seq Datasets } \\
\hline \multicolumn{5}{|c|}{ Helicobacter pylori infection } \\
\hline \multirow{2}{*}{\multicolumn{5}{|c|}{ Helicobacter pylori infected $G C$}} \\
\hline & & & & \\
\hline \multicolumn{5}{|c|}{ Gastric cancer } \\
\hline GSE41476 & Illumina Genome Analyzer II & 5 (3 tumor \& 2 normal $)$ & South Korea & 23 \\
\hline GSE80388 & Illumina MiSeq & 8 (6 tumor $\& 2$ normal $)$ & Taiwan & 24 \\
\hline \multicolumn{5}{|c|}{ Gastritis } \\
\hline E-MTAB-3689 & Illumina HiScan 2500 & $10(6$ tumor $\& 4$ normal $)$ & Nicaragua & 25 \\
\hline
\end{tabular}

Table 2. Differentially expressed genes of H.pylori infection, gastritis, H.pylori infected GC datasets

\begin{tabular}{cc}
\hline \multicolumn{2}{c}{ Microarray Analysis } \\
\hline ID & Number of obtained DEGs \\
\hline E-GEOD-5081 & 254 \\
E-GEOD-74577 & 2198 \\
E-GEOD-74577 & 483 \\
E-GEOD-19826 & 920 \\
E-GEOD-49051 & 6146 \\
E-GEOD-79973 & 1898 \\
\hline \multicolumn{2}{c}{ RNA-seq Analysis } \\
\hline GSE55699 & 908 \\
GSE73226 & 2424 \\
E-MTAB-3689 & 314 \\
GSE73226 & 2066 \\
GSE41476 & 1548 \\
\hline
\end{tabular}


A

\section{E-GEOD-5081}

EnhancedVolcano

NS $\log _{2} F C \bigcirc p$-value $\bigcirc$-value and $\log _{2}$ FC
B

\section{E-GEOD-70394}

EnhancedVolcano

NS $\log _{2} F C \bigcirc p$-value $p$-value and $\log _{2} F C$

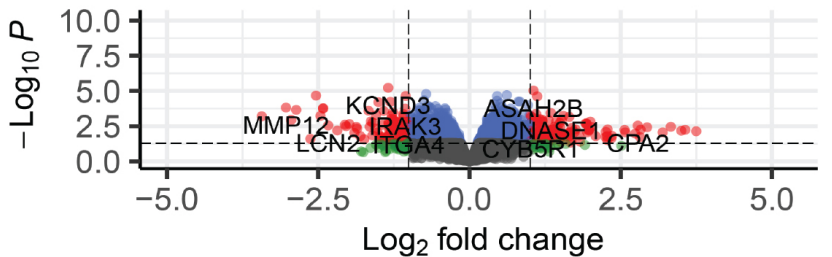

C

\section{E-GEOD-49051}

EnhancedVolcano

NS $\log _{2}$ FC $\bigcirc$-value $p$-value and $\log _{2}$ FC

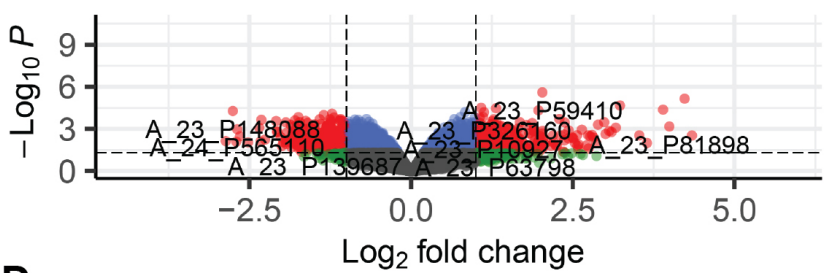

D

\section{E-GEOD-19826}

EnhancedVolcano

NS $\log _{2} F C \bigcirc p$-value $p$-value and $\log _{2}$ FC

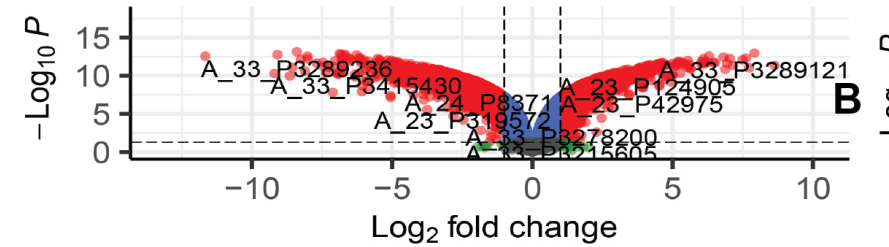

$\mathbf{E}$

total $=34183$ variables

\section{E-GEOD-74577}

EnhancedVolcano

NS $\log _{2} F C \bigcirc p$-value $p$-value and $\log _{2}$ FC

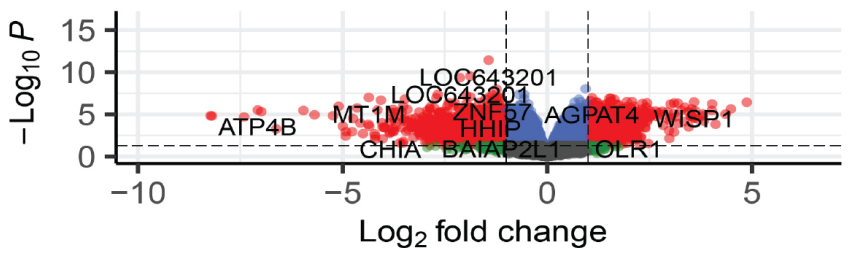

total $=54675$ variables

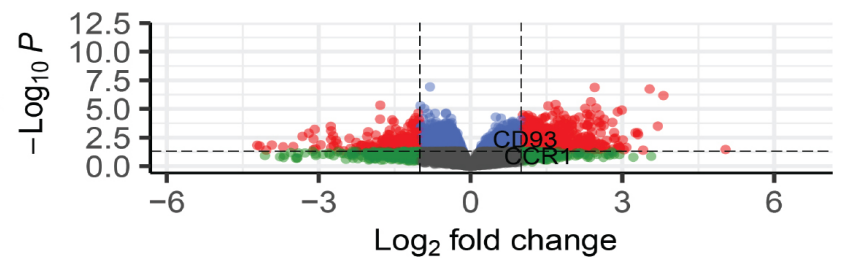

$\mathbf{F}$

\section{E-GEOD-79973}

EnhancedVolcano

NS $\log _{2} F C \bigcirc p$-value $p$-value and $\log _{2} F C$

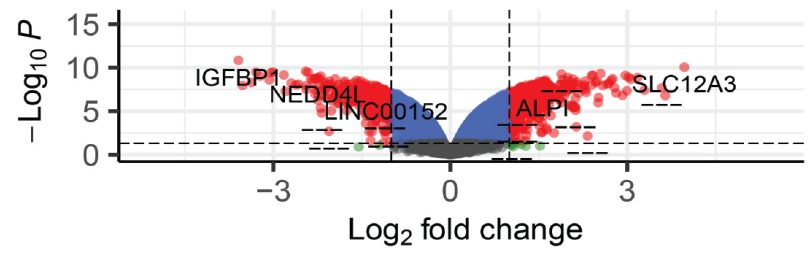

total $=69994$ variables

Figure 2. Differential expression analysis of microarray datasets (H.pylori infection and GC datasets): Enhanced volcano plots for H.pylori infection and GC datasets; A: Dataset E-GEOD-5081 B: Dataset E-GEOD-70394 C: Dataset E-GEOD-74577 D: Dataset E-GEOD-19826 E: Dataset E-GEOD-49051 F: Dataset E-GEOD-79973. In the volcano plots, on the $\mathrm{x}$-axis $\log _{2} \mathrm{FC}$ i.e. $\log _{2} \mathrm{FC}<-1$ and $\log _{2} \mathrm{FC}>1$ was plotted against negative $\log$ of p.value $<0.05$ on the $\mathrm{y}$-axis. Here, the red dots represents the genes that are significantly differentially expressed. The right side of plots shows the up-regulated DEGs while the DEGs plotted on the left side are down-regulated. The blue dots display gene that are significant but not differentially expressed and the green dots are genes that are differentially expressed but not significant. Whereas, the gray dots represents genes that are neither significant nor differentially expressed. 
A

GSE55699

EnhancedVolcano

NS $\bigcirc \log _{2} F C \bigcirc p$-value $p$-value and $\log _{2}$ FC

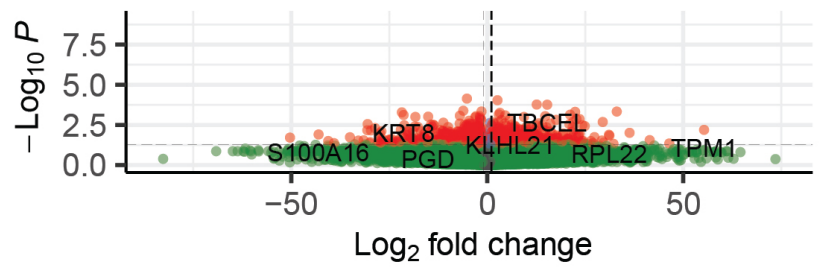

total $=16172$ variables

\section{C}

GSE41476

EnhancedVolcano

NS $\log _{2}$ FC $p$-value $p$-value and $\log _{2}$ FC

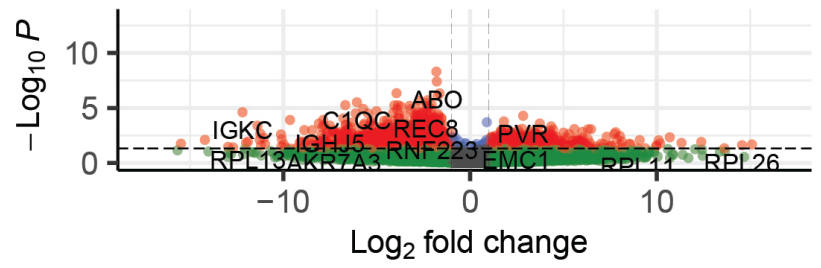

total $=21882$ variables

$\mathbf{E}$

E-MTAB_3689
B

GSE73226

EnhancedVolcano

NS $\log _{2} \mathrm{FC} \bigcirc \mathrm{p}$-value $\mathrm{p}$-value and $\log _{2} \mathrm{FC}$

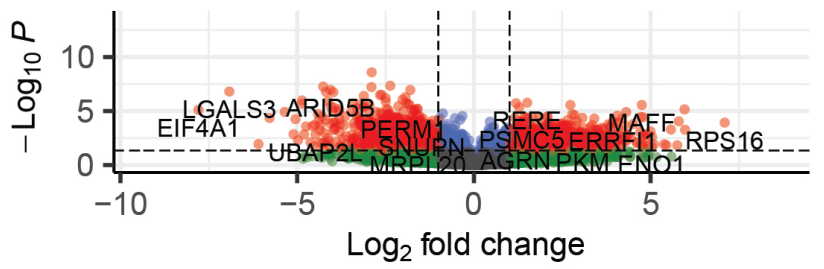

total $=17732$ variables

D

\section{GSE80388}

EnhancedVolcano

NS $\log _{2} F C \bigcirc p$-value $p$-value and $\log _{2} F C$

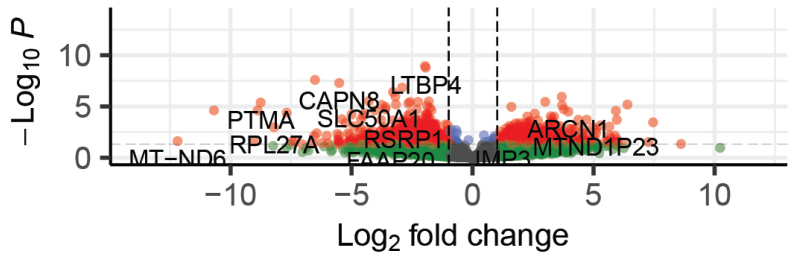

total $=13789$ variables

EnhancedVolcano

NS $\log _{2} \mathrm{FC} \bigcirc \mathrm{p}$-value $\mathrm{p}$-value and $\log _{2} \mathrm{FC}$

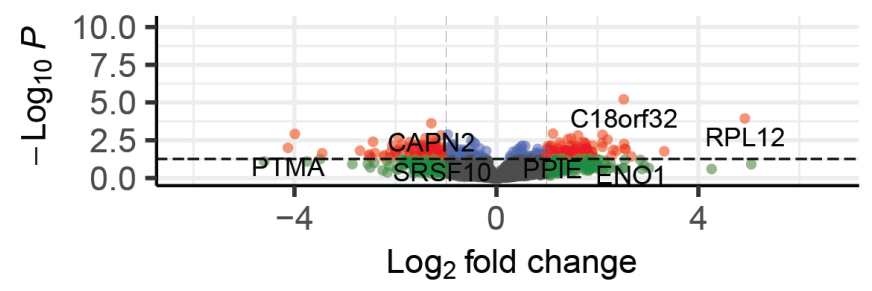

total $=9977$ variables

Figure 3. Differential expression analysis of RNA-seq datasets (H.pylori infection, gastritis, H.pylori infected GC and GC datasets): Enhanced volcano plots for H.pylori infection, gastritis, H.pylori infected GC and GC datasets; A: Dataset GSE55699 B: Dataset GSE73226 C: Dataset GSE41476 D: Dataset GSE80388 E: Dataset E-MTAB-3689, with $\log _{2}$ FC $<-1$ and $\log _{2} \mathrm{FC}>1$ and its p.value $<0.05$. Here, the red dots represents the significant DEGs where on the right side of the plot are up-regulated DEGs and on the left side are the down-regulated DEGs. The blue dots display genes that are significant but not differentially expressed and the green dots are genes that are differentially expressed but not significant. Whereas, the gray dots represents genes that are neither significant nor differentially expressed. 
A

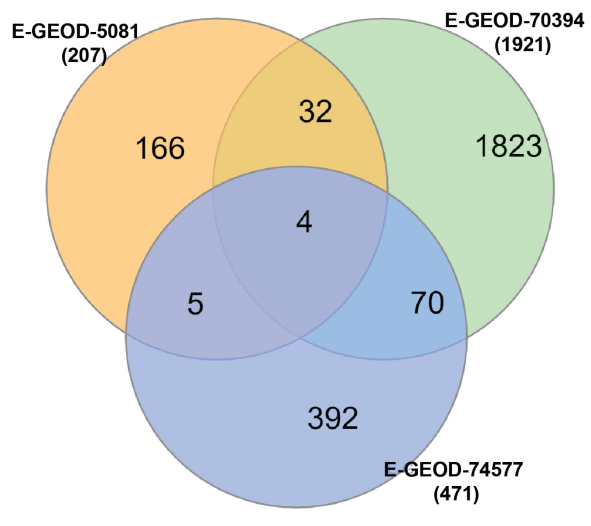

B

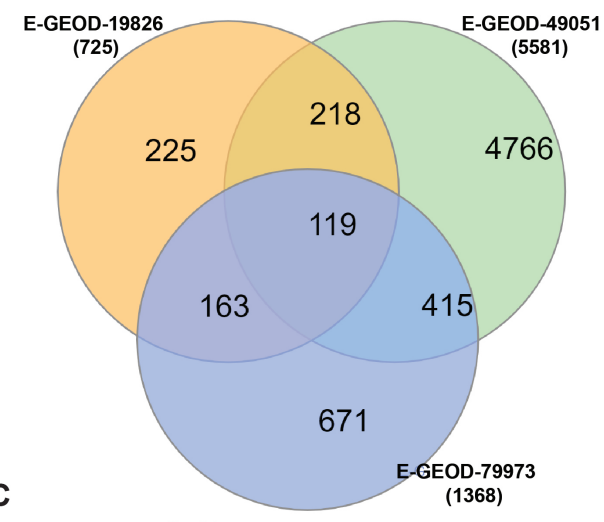

Gastric cancer

(119)

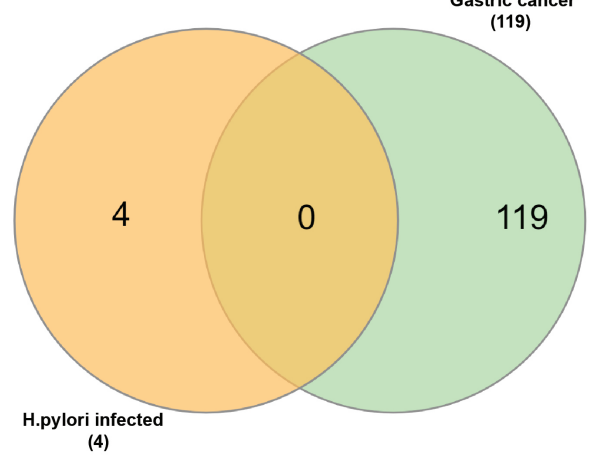

(4)

Figure 4. Comparative analysis-Microarray datasets: A: From H.pylori infection datasets 4 common DEGs identified; B: From GC datasets 119 common DEGs identified; C: No common DEGs identified from integrative analysis of H.pylori infection and GC microarray datasets. 


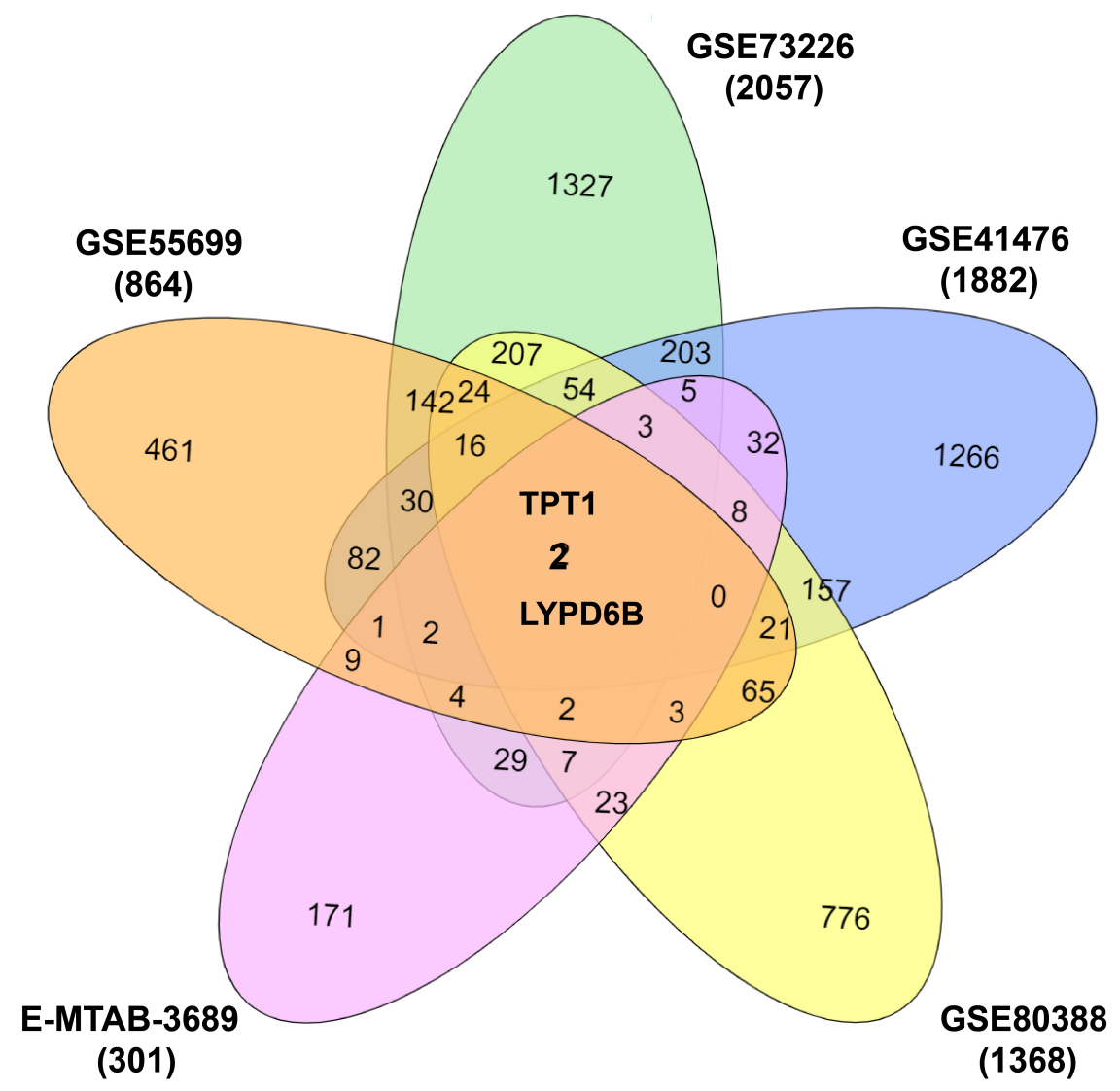

Figure 5. Comparative analysis-RNA-seq datasets: A: From integrating the DEGs from H.pylori infection,gastritis, H.pylori infected GC and GC, we identified 2 common DEGs (TPT1 and LYPD6B). 


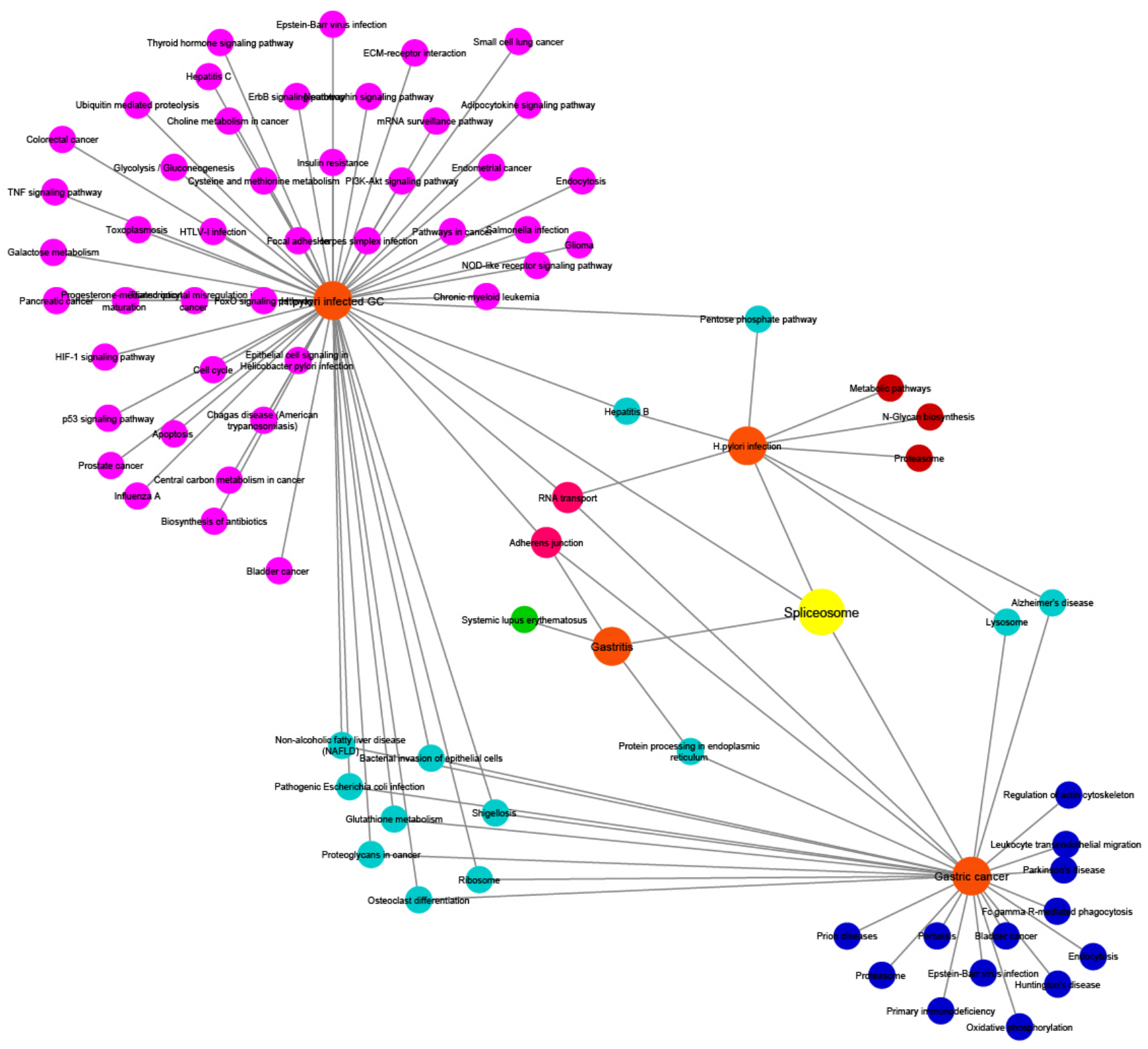

Figure 6. Pathway interactions in H.pylori infection, gastritis, H.pylori infected GC and GC: This network shows the common interactive pathways involved in the four conditions. The yellow node shows spliceosome which is found to be the sole common pathway across all the conditions, whereas, the 2 pink nodes (Adherens junction and RNA transport) were the pathways common in three conditions. Other common pathways are given light blue color based on interactions in two conditions. 

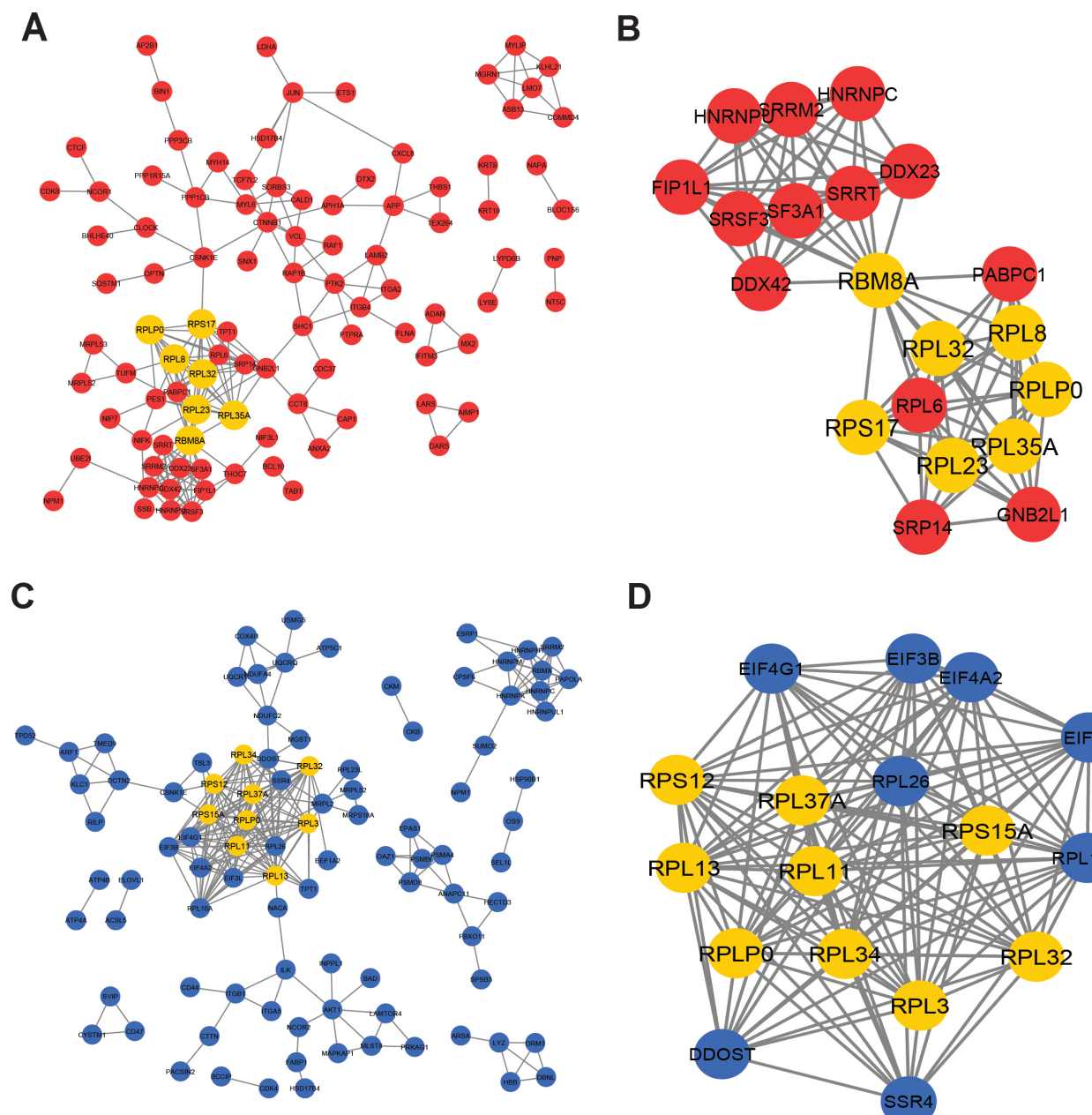

D
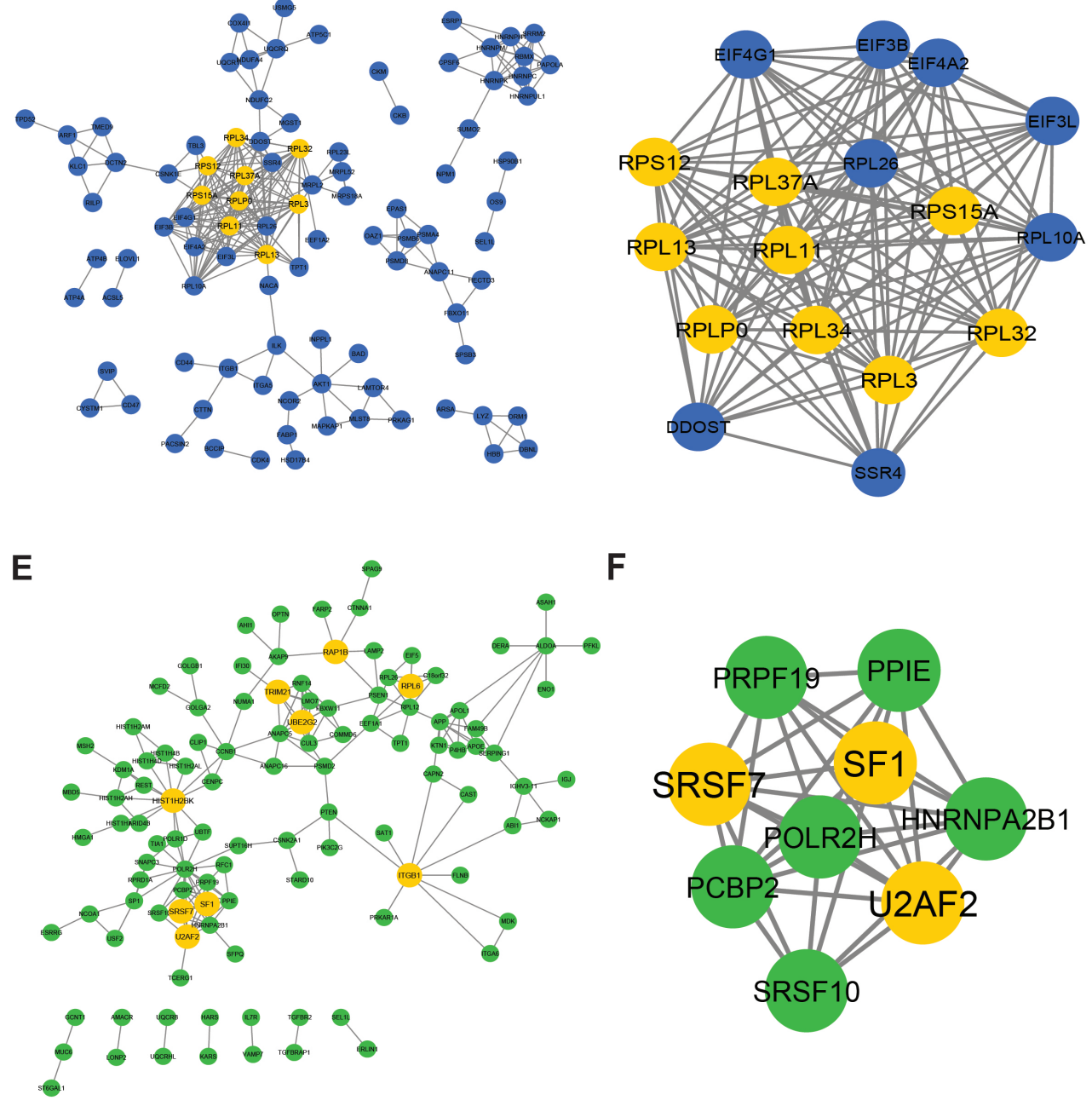

$\mathbf{F}$

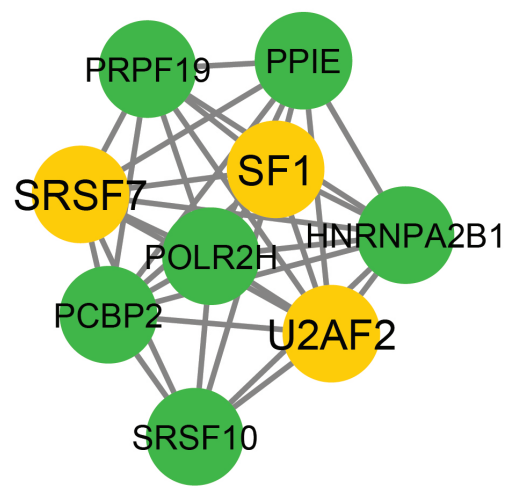

Figure 7. Hub genes in H.pylori infection, gastritis, H.pylori infected GC and GC protein-protein interaction (PPI) networks: A: PPI of H.pylori infection indicating 17 hub genes along with highly interactive genes cluster B: PPI network of gastritis indicating 9 hub genes with a cluster of highly interactive genes in the network. C: PPI network of $H$.pylori infected GC indicating 14 hub genes with a cluster of highly interactive genes in the network. D: PPI network of GC indicating 9 hub genes along with a highly interactive genes cluster 


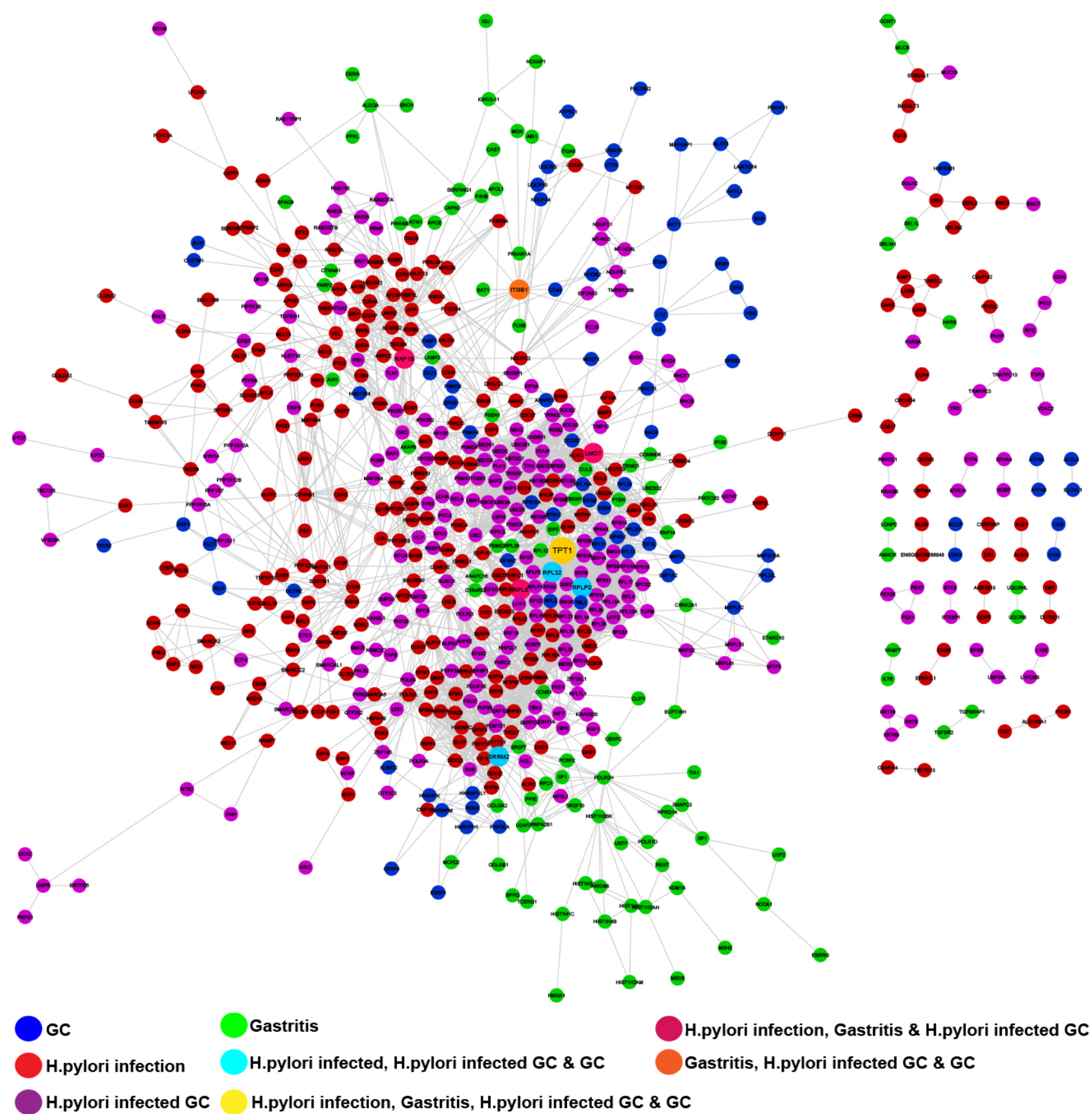

Figure 8. Common interactions and potential markers in H.pylori infection, gastritis, H.pylori infected GC and GC: Network indicates common significant interactions in H.pylori infection, gastritis, H.pylori infected GC and GCH.pylori infection, gastritis, H.pylori infected GC and GC. Here TPT1 gene is common in all the conditions. 
Table 3. Top enriched ontologies retrieved from Toppgene

\begin{tabular}{|c|c|c|c|c|c|}
\hline ID & Function & Name & P.Value & FDR & Entities \\
\hline \multicolumn{6}{|c|}{ H.pylori infection enriched ontologies } \\
\hline GO:0003723 & MF & RNA binding & $1.16 \mathrm{E}-08$ & $1.69 \mathrm{E}-05$ & 135 \\
\hline GO:0045296 & MF & Cadherin binding & $1.39 \mathrm{E}-07$ & $1.02 \mathrm{E}-04$ & 37 \\
\hline GO:0050839 & MF & Cell adhesion molecule binding & $1.23 \mathrm{E}-06$ & $6.00 \mathrm{E}-04$ & 47 \\
\hline GO:0044265 & BP & Cellular macromolecule catabolic process & 8.78E-09 & $5.55 \mathrm{E}-05$ & 90 \\
\hline GO:0019752 & $\mathrm{BP}$ & Carboxylic acid metabolic process & $1.77 \mathrm{E}-08$ & $5.55 \mathrm{E}-05$ & 85 \\
\hline GO:0006082 & $\mathrm{BP}$ & Organic acid metabolic process & $3.66 \mathrm{E}-08$ & $5.55 \mathrm{E}-05$ & 90 \\
\hline GO:0005925 & $\mathrm{CC}$ & Focal adhesion & $5.60 \mathrm{E}-10$ & 5.33E-07 & 45 \\
\hline GO:0030055 & $\mathrm{CC}$ & Cell -substrate junction & $1.83 \mathrm{E}-09$ & 8.73E-07 & 109 \\
\hline GO:0005912 & $\mathrm{CC}$ & Adherens junction & $1.02 \mathrm{E}-08$ & $2.53 \mathrm{E}-06$ & 93 \\
\hline \multicolumn{6}{|c|}{ Gastritis enriched ontologies } \\
\hline GO:0003823 & MF & Antigen binding & $8.63 \mathrm{E}-09$ & $6.95 \mathrm{E}-06$ & 15 \\
\hline GO:0003723 & MF & RNA binding & $9.99 \mathrm{E}-06$ & $2.68 \mathrm{E}-03$ & 49 \\
\hline GO:0050839 & MF & Cell adhesion molecule binding & $7.46 \mathrm{E}-05$ & $1.20 \mathrm{E}-02$ & 19 \\
\hline GO:0006958 & BP & Complement activation, classical pathway & $3.05 \mathrm{E}-12$ & $1.38 \mathrm{E}-08$ & 17 \\
\hline GO:0002455 & BP & Humoral immune response & $1.48 \mathrm{E}-11$ & $3.35 \mathrm{E}-08$ & 17 \\
\hline GO:0030449 & BP & Regulation of complement activation & $2.94 \mathrm{E}-11$ & $3.48 \mathrm{E}-08$ & 15 \\
\hline GO:0019814 & $\mathrm{CC}$ & Immunoglobulin complex & $2.09 \mathrm{E}-14$ & $1.35 \mathrm{E}-11$ & 20 \\
\hline GO:1902494 & $\mathrm{CC}$ & Catalytic complex & $3.05 \mathrm{E}-06$ & $9.84 \mathrm{E}-04$ & 42 \\
\hline GO:1990234 & $\mathrm{CC}$ & Transferase complex & $6.83 \mathrm{E}-05$ & $1.47 \mathrm{E}-02$ & 25 \\
\hline \multicolumn{6}{|c|}{ H.pylori infected enriched ontologies } \\
\hline GO:0045296 & MF & Cadherin binding & $9.56 \mathrm{E}-16$ & $1.61 \mathrm{E}-11$ & 82 \\
\hline GO:0003723 & MF & RNA binding & $6.68 \mathrm{E}-15$ & $5.61 \mathrm{E}-11$ & 289 \\
\hline GO:0050839 & MF & Cell adhesion molecule binding & $1.73 \mathrm{E}-14$ & $9.70 \mathrm{E}-11$ & 107 \\
\hline GO:0016032 & $\mathrm{BP}$ & Viral process & $2.32 \mathrm{E}-32$ & $2.12 \mathrm{E}-27$ & 189 \\
\hline GO:0044403 & $\mathrm{BP}$ & Symbiotic process & $2.84 \mathrm{E}-31$ & $1.30 \mathrm{E}-26$ & 195 \\
\hline GO:0044419 & $\mathrm{BP}$ & Interspecies interaction between organisms & $5.84 \mathrm{E}-30$ & $1.78 \mathrm{E}-25$ & 199 \\
\hline GO:0030055 & $\mathrm{CC}$ & Cell-substrate junction & $2.95 \mathrm{E}-32$ & $2.09 \mathrm{E}-28$ & 121 \\
\hline GO:0005925 & $\mathrm{CC}$ & Focal adhesion & $4.54 \mathrm{E}-32$ & $2.09 \mathrm{E}-28$ & 119 \\
\hline GO:0005912 & $\mathrm{CC}$ & Adherens junction & $1.07 \mathrm{E}-30$ & $3.28 \mathrm{E}-27$ & 141 \\
\hline \multicolumn{6}{|c|}{ GC enriched ontologies } \\
\hline GO:0003735 & $\mathrm{MF}$ & Structural constituent of ribosome & $3.89 \mathrm{E}-19$ & $9.50 \mathrm{E}-16$ & 78 \\
\hline GO:0003723 & MF & RNA binding & $2.43 \mathrm{E}-16$ & $5.93 \mathrm{E}-13$ & 285 \\
\hline GO:0050839 & MF & Cell adhesion molecule binding & $5.98 \mathrm{E}-13$ & $1.46 \mathrm{E}-09$ & 130 \\
\hline GO:0044403 & BP & Symbiotic process & $1.35 \mathrm{E}-33$ & $1.41 \mathrm{E}-29$ & 249 \\
\hline GO:0016032 & BP & Viral process & $3.64 \mathrm{E}-33$ & $3.82 \mathrm{E}-29$ & 237 \\
\hline GO:0044419 & BP & Interspecies interactions between organisms & $3.16 \mathrm{E}-32$ & $3.16 \mathrm{E}-32$ & 255 \\
\hline GO:0044391 & $\mathrm{CC}$ & Ribosomal subunit & $1.04 \mathrm{E}-29$ & $1.41 \mathrm{E}-26$ & 94 \\
\hline GO:0005739 & $\mathrm{CC}$ & Mitochondrion & $2.36 \mathrm{E}-28$ & $3.20 \mathrm{E}-25$ & 399 \\
\hline GO:0019814 & $\mathrm{CC}$ & Immunoglobulin complex & $3.63 \mathrm{E}-28$ & $4.93 \mathrm{E}-25$ & 77 \\
\hline
\end{tabular}


Table 4. KEGG pathways retrieved from DAVID

\begin{tabular}{|c|c|c|c|}
\hline KEGG ID & Term & Count & P.Value \\
\hline \multicolumn{4}{|c|}{ H.pylori infection enriched KEGG pathways } \\
\hline hsa03040 & Spliceosome & 16 & $3.13 \mathrm{E}-03$ \\
\hline hsa01100 & Metabolic pathways & 80 & $1.20 \mathrm{E}-02$ \\
\hline hsa03050 & Proteasome & 7 & $2.34 \mathrm{E}-02$ \\
\hline hsa05010 & Alzheimer's disease & 16 & $2.47 \mathrm{E}-02$ \\
\hline hsa05161 & Hepatitis B & 14 & $3.41 \mathrm{E}-02$ \\
\hline \multicolumn{4}{|c|}{ Gastritis enriched KEGG pathways } \\
\hline hsa04520 & Adherens junction & 6 & 0.004976 \\
\hline hsa03040 & Spliceosome & 7 & 0.017902 \\
\hline hsa05322 & Systemic lupus erythematosus & 7 & 0.018509 \\
\hline hsa04141 & Protein processing in endoplasmic reticulum & 7 & 0.049395 \\
\hline \multicolumn{4}{|c|}{ H.pylori infected Gastric Cancer enriched KEGG pathways } \\
\hline hsa03010 & Ribosome & 48 & $8.56 \mathrm{E}-13$ \\
\hline hsa03013 & RNA transport & 43 & $1.32 \mathrm{E}-06$ \\
\hline hsa04510 & Focal adhesion & 44 & $6.62 \mathrm{E}-05$ \\
\hline hsa05131 & Shigellosis & 20 & $6.81 \mathrm{E}-05$ \\
\hline hsa03040 & Spliceosome & 24 & $3.00 \mathrm{E}-02$ \\
\hline \multicolumn{4}{|c|}{ Gastric Cancer enriched KEGG pathways } \\
\hline hsa03010 & Ribosome & 68 & $3.15 \mathrm{E}-19$ \\
\hline hsa05016 & Huntington's disease & 59 & $1.26 \mathrm{E}-06$ \\
\hline hsa03013 & RNA transport & 54 & $1.87 \mathrm{E}-06$ \\
\hline hsa05010 & Alzheimer's disease & 49 & $5.19 \mathrm{E}-05$ \\
\hline hsa03040 & Spliceosome & 35 & 0.004784 \\
\hline
\end{tabular}

Table 5. Top hub genes in H.pylori infection, gastritis, H.pylori infected GC and GC protein-protein interaction networks

\begin{tabular}{|c|c|c|c|c|c|c|c|}
\hline Gene & Avg. Shortest Path Length & Betweenness Centrality & Closeness Centrality & Clustering Coefficient & Degree & Eccentricity & Neighborhood Connectivity \\
\hline \multicolumn{8}{|c|}{ H.pylori infection Hub genes } \\
\hline RPS20 & 1.375 & 0.282710091 & 0.727272727 & 0.234482759 & 24 & 3 & 19.8 \\
\hline RPLP0 & 1.4 & 0.060595847 & 0.714285714 & 0.316239316 & 21 & 2 & 21.2962963 \\
\hline RPS17 & 1.444444444 & 0.181880964 & 0.692307692 & 0.276353276 & 21 & 3 & 21 \\
\hline SRSF3 & 1 & 0.067611955 & 1 & 0.349802372 & 20 & 1 & 19.47826087 \\
\hline RPL8 & 1.363636364 & 0.087170271 & 0.733333333 & 0.307692308 & 20 & 2 & 21.18518519 \\
\hline POLR2L & 1.844444444 & 2.734273198 & 0.542168675 & 0.132132132 & 19 & 4 & 13.05405405 \\
\hline SENP3 & 1 & 0.208449878 & 1 & 0.223684211 & 18 & 1 & 18.55 \\
\hline RPL6 & 1.384615385 & 0.067164488 & 0.722222222 & 0.329230769 & 18 & 2 & 21.76923077 \\
\hline SRRT & 1.4 & 0.030429322 & 0.714285714 & 0.371428571 & 18 & 2 & 19.61904762 \\
\hline RNPS1 & 1.4 & 0.82547346 & 0.714285714 & 0.211111111 & 17 & 3 & 21.16666667 \\
\hline \multicolumn{8}{|c|}{ Gastritis Hub genes } \\
\hline SRSF7 & 3.036082474 & 0.02984606 & 0.329371817 & 0.470588235 & 17 & 6 & 12.11764706 \\
\hline $\mathrm{U} 2 \mathrm{AF} 2$ & 3.139175258 & 0.014312771 & 0.318555008 & 0.558333333 & 16 & 6 & 13.0625 \\
\hline ITGB1 & 3.139175258 & 0.099231964 & 0.318555008 & 0.058333333 & 16 & 7 & 4.375 \\
\hline SF1 & 3.077319588 & 0.022461931 & 0.324958124 & 0.628571429 & 15 & 6 & 13.6 \\
\hline TRIM21 & 3.170103093 & 0.058294451 & 0.315447154 & 0.272727273 & 11 & 7 & 7.090909091 \\
\hline HIST1H2AH & 3.484536082 & 0.017094311 & 0.286982249 & 0.563636364 & 11 & 8 & 9.545454545 \\
\hline RPL6 & 3.242268041 & 0.011645183 & 0.308426073 & 0.436363636 & 11 & 6 & 10.90909091 \\
\hline UBE2G2 & 3.164948454 & 0.03519891 & 0.315960912 & 0.377777778 & 10 & 7 & 8.9 \\
\hline RAP1B & 3.365979381 & 0.030676355 & 0.297090352 & 0.095238095 & 7 & 8 & 7 \\
\hline \multicolumn{8}{|c|}{ H.pylori infected Gastric cancer Hub genes } \\
\hline UPF2 & 0 & 0 & 0 & 0.5 & 42 & 0 & 49.5 \\
\hline UPF1 & 1 & 0 & 1 & 0.5 & 41 & 1 & 49.5 \\
\hline SSR2 & 0 & 0 & 0 & 0.497560976 & 41 & 0 & 48.41463415 \\
\hline SMG6 & 1 & 0 & 1 & 0.5 & 40 & 1 & 49.5 \\
\hline RPS9 & 1 & 0.038577629 & 1 & 0.426470588 & 39 & 1 & 45.63461538 \\
\hline RPS7 & 1.071428571 & 0.16607394 & 0.933333333 & 0.422699849 & 39 & 2 & 45.71153846 \\
\hline SMG1 & 1 & 0 & 1 & 0.5 & 39 & 1 & 49.5 \\
\hline RPS4X & 1.066666667 & 0.16607394 & 0.9375 & 0.422699849 & 38 & 2 & 45.71153846 \\
\hline SPCS2 & 1 & 0 & 1 & 0.5 & 38 & 1 & 48.94871795 \\
\hline SRP9 & 1 & 0 & 1 & 0.5 & 38 & 1 & 48.94871795 \\
\hline \multicolumn{8}{|c|}{ GC Hub genes } \\
\hline RPS12 & 2.854545455 & 0.128086055 & 0.350318471 & 0.707602339 & 19 & 7 & 14.26315789 \\
\hline RPS15A & 2.854545455 & 0.128086055 & 0.350318471 & 0.707602339 & 19 & 7 & 14.26315789 \\
\hline RPL13 & 2.618181818 & 0.455179209 & 0.381944444 & 0.713450292 & 19 & 6 & 14.42105263 \\
\hline RPL32 & 2.909090909 & 0.05652601 & 0.34375 & 0.713450292 & 19 & 7 & 14.36842105 \\
\hline RPL11 & 2.927272727 & 0.020162374 & 0.341614907 & 0.797385621 & 18 & 7 & 15.16666667 \\
\hline RPL3 & 3 & 0.004180331 & 0.333333333 & 0.867647059 & 17 & 7 & 15.64705882 \\
\hline RPL34 & 2.945454545 & 0.017532905 & 0.339506173 & 0.852941176 & 17 & 7 & 15.70588235 \\
\hline RPL37A & 2.945454545 & 0.017532905 & 0.339506173 & 0.852941176 & 17 & 7 & 15.70588235 \\
\hline RPLP0 & 3 & 0.004180331 & 0.333333333 & 0.867647059 & 17 & 7 & 15.64705882 \\
\hline
\end{tabular}


Table 6. Networks summary statistics of H.pylori infection, gastritis H.pylori infected GC and GC protein-protein interaction networks

\begin{tabular}{cccccc}
\hline Network & Nodes & Edges & Connected components & Avg. number of neighbours & Clustering coefficient \\
\hline H.pylori infection & 259 & 388 & 20 & 2.9 & 0.107 \\
H.pylori infected Gastric cancer & 136 & 1956 & 1 & 28.76 & 0.42 \\
Gastric cancer & 96 & 263 & 10 & 5.47 & 0.284 \\
Gastritis & 112 & 195 & 8 & 3.48 & 0.205 \\
\hline
\end{tabular}




\section{Supplementary Files}

This is a list of supplementary files associated with this preprint. Click to download.

- Pathways.xlsx

- RNAseqDifferentiallyExpressedGenes.xlsx 